\title{
Self-tuning digital Mössbauer detection system
}

\author{
A. Veiga ${ }^{*, 1,2}$, C.M. Grunfeld ${ }^{1,2,3}$, G.A. Pasquevich ${ }^{1,2,3}$, P. Mendoza Zélis ${ }^{1,2,3}$, N. \\ Martínez ${ }^{2,3,4}$ and F.H. Sánchez ${ }^{2,3}$ \\ ${ }^{1}$ Facultad de Ingeniería, Universidad Nacional de La Plata, Argentina \\ ${ }^{2}$ Instituto de Física La Plata, CONICET, Argentina \\ ${ }^{3}$ Facultad de Cs. Exactas, Universidad Nacional de La Plata, Argentina \\ ${ }^{4}$ Comisión de Investigaciones Científicas de la Provincia de Buenos Aires, \\ Argentina \\ * Corresponding author \\ email: alejandro.veiga@ing.unlp.edu.ar \\ Phone/FAX (54) 221-4259306
}

\begin{abstract}
Long term gamma spectroscopy experiments involving single-channel analyzer equipment depend upon thermal stability of the detector and its associated high-voltage supply. Assuming constant discrimination levels, a drift in the detector gain impacts the output rate, producing an effect on the output spectrum. In some cases (e.g. single-energy resonant absorption experiments) data of interest can be completely lost. We present a digital self-adapting discrimination strategy that tracks emission line shifts using statistical measurements on a predefined region-of-interest of the spectrum. It is developed in the form of a synthesizable module that can be intercalated in the digital processing chain. It requires a moderate to small amount of digital resources and can be easily activated and deactivated.
\end{abstract}

Keywords Mössbauer - Instrumentation - Spectrometer - SCA - Constant-velocity 


\section{Introduction}

A Mössbauer detection system is a classical single energy selector operating on low energy gamma events [1-3]. It is composed of four main elements: a proportional counter with high-voltage (HV) supply, a charge preamplifier, a shaping amplifier and a single channel analyzer (SCA), as shown in Fig. 1A. Long term stability of the system is an important factor, especially in high resolution experiments, being critical in constant-velocity applications [4-9]. In these cases transmission is recorded at a single energy for a long period of time. Under unstable conditions, an output rate variation cannot be distinguished to be the effect of the resonant process under study or a consequence of a shift in spectrometer operating point.

The primary source of long term instability is temperature dependence of both the HV-supply and the gaseous detector gain [10]. A temperature change displaces the emission line out of the SCA window center, changing the proportion between resonant and non-resonant photons. In precision single energy experiments either temperature control must be implemented or a reference background rate must be periodically recorded, with a consequent loss in efficiency.

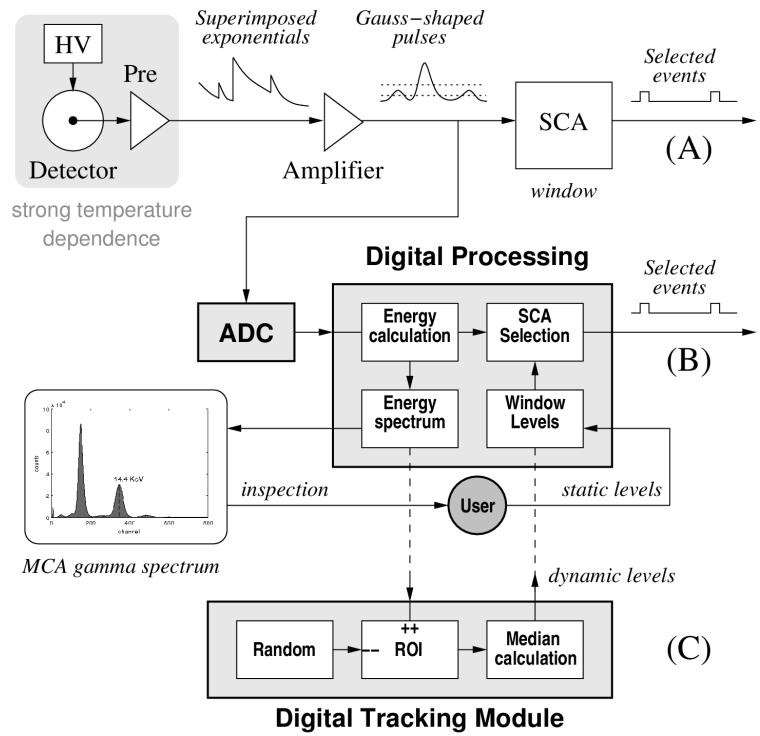

Fig. 1 Mössbauer detection system composed of proportional counter and preamplifier, followed by: (A) shaping amplifier and single channel analyzer (SCA) in analog classical NIM layout, or (B) analog-to-digital converter (ADC) and digital processing module in digitized version. User interaction for window selection is schematized. The proposed tracking module (C) can be designed to coexist with previous configuration.

Digitization of the detection system provides a powerful environment where a compensation algorithm for temperature drifts can be devised. In a regular digital arrangement, as shown in Fig. 1B, output of the shaping amplifier is sampled by an analog-to-digital converter (ADC) and sent to a digital processing module. Every detected event is digitized and translated into energy by a shaping algorithm. A digital output is generated if SCA selection criteria on energy is met (e.g. amplitude window). The selection criterion is provided by user after initial 
full spectrum inspection (MCA) and remains unaltered during the experiment. This setup is well addressed in bibliography [11-15] and several FPGA implementations have proved to be successful.

We will show how, with a moderate amount of additional digital resources, the processing module can be extended to automatically monitor the status of the emission spectrum, detect potential temperature drifts and adjust the selection criteria, in order to keep a constant background rate. The proposed tracking module, shown in Fig. 1C, can be enabled or disabled as required. When disabled, the fixed window criterion initially selected by the user can be applied.

In the next sections a window-based tracking strategy design is presented and the main implementation aspects of the proposed techniques are discussed. Experimental results illustrate the performance of the tracking module when perturbations are induced in the detection system.

\section{Proposed tracking technique}

As a first approximation, it can be assumed that the thermal drift in the detector will only displace the emission line center $\left(14.4 \mathrm{KeV}\right.$ in ${ }^{57} \mathrm{Fe}$ Mössbauer experiments), keeping its variance unaltered. In consequence, a fixed width, moving energy window can provide a simple way to implement an adaptive selection criterion. Then, the tracking problem is reduced to find the proper displacement of the window, which center will be coincident with the actual emission line center. Fig. 2 shows the behavior of a section of ${ }^{57} \mathrm{Fe}$ source spectrum when a $10 \%$ change in amplifier gain is forced.

We define the tracking window as the range of spectrum channels comprised between $\mathrm{C}-\mathrm{W}$ and $\mathrm{C}+\mathrm{W}$, being $\mathrm{C}$ the window central channel and $\mathrm{W}$ the window half-width. $\mathrm{C}$ is designed to be initially set by user inspection of the whole spectrum and afterward continuously relocated by the tracking algorithm. W remains unaltered during the tracking process, being advisable to be set to the optimal value for the emission line $[16,17]$.

The region inside which the window can be moved is defined as the region of interest (ROI) of the digital processing module. This region (a fixed subset of channels larger than the window) can be selected upon experimental evidence. An empirical value of twice the window can be used, from channel $\mathrm{C}-2 \mathrm{~W}$ to $\mathrm{C}+2 \mathrm{~W}$. The ROI spectrum is continuously updated by the digital processing module. An algorithm is applied to the window section in order to locate the emission line properties. Using this information, window levels are dynamically adjusted inside the ROI. The resulting center is used to compensate SCA discrimination levels.

For example, given a 12-bit digitizer (4096 levels) a 10-bit spectrum (1024 channels) can be built and a 7-bit ROI (128 channels) can be selected. Inside it, a 6-bit window (64 channels) can shift. In Fig. 2, this window tracks the $14.4 \mathrm{KeV}$ line inside the static ROI, from channel $\mathrm{C} 1$ to channel $\mathrm{C} 2$. 

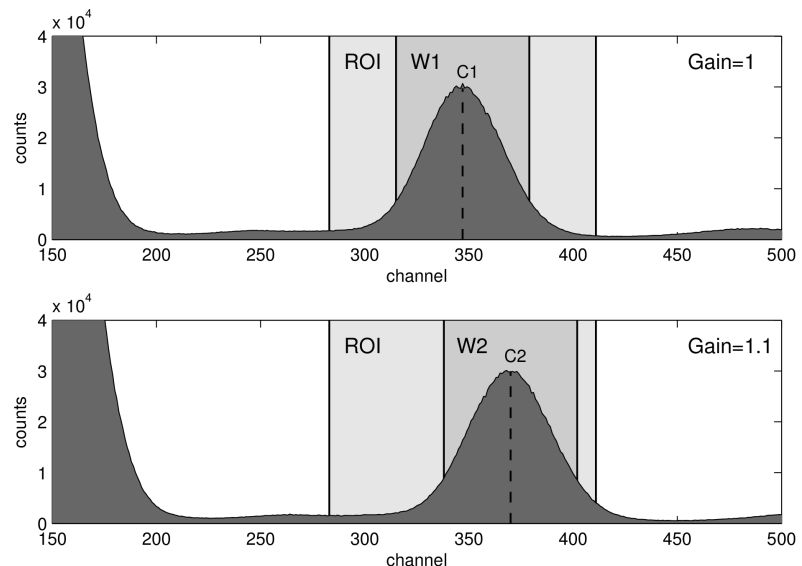

Fig. 2 Section of an experimental ${ }^{57} \mathrm{Fe}$ source MCA spectrum (1024 channels), showing fixed 7-bit ROI (128 channels) and tracking 6-bit window (64 channels), when a $10 \%$ increase in amplifier gain is forced. The window tracks the $14.4 \mathrm{KeV}$ line inside the ROI, from channel $\mathrm{C} 1$ to channel $\mathrm{C} 2$.

Under these circumstances, digital implementation of the tracking strategy presents two main challenges:

i) In order to keep updated information that can be aware of changes, ROI must be stored and continuously populated with the newly detected events, while the older ones are discarded.

ii) A new window center must be recalculated every time ROI is updated, using stored information and an algorithm capable of being implemented with medium complexity digital resources.

These two subjects are addressed in detail in next two sections.

\section{ROI storage and update}

Given the characteristics of the low level trigger algorithm (Energy Calculation module in Fig. 1), the tracking strategy is designed on an event-based operation. Its input is the processed energy of the newly detected gamma event, provided by the low level trigger. This event is used to update ROI and to initiate window recalculation. In order to keep stored ROI information updated, two different strategies can be considered.

A simplistic approach would be to record the gamma spectrum for a fixed period of time (enough to reduce statistical uncertainty, collecting $N$ events), use it to calculate the new window center, and reset the spectrum to start a new acquisition period. However, that may result in a slow tracking response because it takes $N /$ rate seconds to get a new spectrum from scratch to calculate the next center.

A more appropriate solution would be to keep ROI updated with the last $N$ detected events, discarding the oldest one every time a new event arrives. A FIFO architecture could be used. Implementation of this technique would result too 'expensive', as it would require the order number to be stored for each of the $N$ events (e.g. for a 256 channel ROI that stores one million events, $1.5 \%$ statistical error/channel, $1 \mathrm{MB}$ of memory is required). Additionally, the algorithm to find 
the center would involve the processing of the full FIFO.

A more efficient stochastic method is proposed: every time a new event arrives, it is stored in the ROI and, immediately, a random event is discarded, keeping ROI area equal to $N$. In order to preserve the spectrum properties, the event to discard must be selected using the probability density function (PDF) of the current spectrum. That can be accomplished by removing a uniformly-distributed random event from the ordered spectrum. Digital implementation of pseudo-random number generators can be addressed using linear feedback shift registers (LFSR) of appropriate length [18].

It is important to note that, to preserve PDF spectrum, it would not be valid to decrement a random channel of the ROI. More exactly, a random event must be discarded, so most populated channels are more likely to be decremented, keeping the spectrum shape unaltered. That can be accomplished by generating an $\mathrm{N}$-bit uniform random number (shifting $N$ times a maximal-length sequence LFSR) [19] and using this number to select one of the $N$ stored events. Is the channel that contains that event the one that must be decremented.

In order to provide storage space for an $M$-channel ROI, capable of collecting $N$ events with increment/decrement functionality, an $M$ array of $\log 2(N)$ up/down counters can be used (256 20-bit counters are required for the previous $1 \mathrm{MB}$ memory example).

\section{Tracking algorithm}

Once the gamma spectrum is stored in ROI and continuously updated, the current emission center can be found by calculating the mean inside the current window limits (these limits were calculated using the previous center). Newly calculated center is used to reposition the window.

If a window of $W$ channels is currently centered in channel $C$, next center channel $C^{\prime}$ can be calculated as

$$
\text { index }_{C^{\prime}}=\frac{\sum_{i=C-W}^{i=C+W} \text { count }_{i} \cdot i}{\sum_{i=C-W}^{i=C+W} \text { count }_{i}}
$$

Being $i$ the channel number and count $_{i}$ the number of events stored in it.

Mean is calculated inside the window limits, not in the whole ROI. That is so because window converges to a narrow (mostly symmetrical) selection of the emission peak. Being much wider, ROI can include spectrum asymmetries that can distort mean calculation (RX lateral peaks, exponential background, etc.). If such asymmetries are not present, widening the window at both sides of the center can improve precision.

Implementing this technique involves an important amount of calculations, and therefore cost, power consumption and dead time. Instead of using first-order moment to find the new center, using the median in the active window can be more efficient since its calculation is simpler and results less dependent of spectrum skewness. Several algorithms are available to calculate median of a 
dataset, most of them involving sorting [20]. That is very convenient, as in our case data is already sorted in the available spectrum.

The proposed tracking technique is not sensitive to the widening (second-order moment) of the dataset, as long as the algorithm is able to find a maximum inside the window. A variance estimator can be implemented on the window channels in order to detect a change, if necessary.

\section{Dynamics and simulations}

The responsiveness of the algorithm will be limited by the amount of events stored in the ROI $(N)$. Such amount is imposed by the required precision in window positioning. Rising it improves window median calculation (statistical uncertainty is reduced), improving the determination of the window center. However, given an event rate $m$, it will take $N / m$ seconds to the algorithm to be fully aware of an abrupt change in rate. Limitations at this point will arise from detector thermal dynamics and HV stability.

Operating parameters can be tuned using simulations in a numerical computing environment (Octave/Matlab® scripts are available). Optimal operating point results from the compromise between responsiveness and precision. For example, considering the case of Fig. 2 (128-channel ROI with 64-channel window and 1M stored events), operating at a rate of $10^{4}$ events/s, it will take at least 100 seconds to fully refresh the ROI.

\section{Algorithm implementation}

Tracking was tested using a development platform comprised of a 16-bit 250 MSPS analog-to-digital converter (AD9467 from Analog Devices) and a Virtex4FX20 FPGA from Xilinx.

Both digital processing and tracking module (all blocks in Fig. 1) were implemented as an event-triggered finite state machine (FSM). USB and UART communication modules were included to download ADC traces, monitor tracking evolution, inspect spectrum and configure parameters at run time. There was enough room in the device to accommodate a 64-channel ROI of 16-bit counters, with a 32-channel window $(\mathrm{N}=64 \mathrm{~K}, \mathrm{M}=64, \mathrm{~W}=16)$. The whole system consumed approximately $90 \%$ of available resources (7,756 of 8,544 slices). It was programmed using a behavioral high-level description language (source code available).

Considering implementation in a more recent Spartan6-LX45 (low-power, lowcost, more advanced FPGA device), the tracking module alone requires 1,707 of 6,822 available logic cells, representing only $25 \%$ of device resources.

\section{Experimental results}

In order to characterize the system, its behavior was tested under different perturbations. Instruments used for the experiment were a proportional counter, a static ${ }^{57} \mathrm{Fe}$ source, an Ortec 142PC preamplifier and an Ortec 572A amplifier 
(Ortec 420A single channel analyzer was used as a reference). The digital selection system was set to operate at a rate of 3,000 events/s.

As an example a fast $14.4 \mathrm{KeV}$ emission line tracking is presented in Fig. 3, where amplifier gain is instantly changed from 55.94 to $56.10(0.3 \%)$. Information of the ROI and the calculated center time evolution were obtained from the digital module through its serial interface. Figure shows an exponential response when line center jumps from ROI channel 18 to 44 (26 channels, outside initial W) in $\mathrm{t}=50 \mathrm{~s}$.

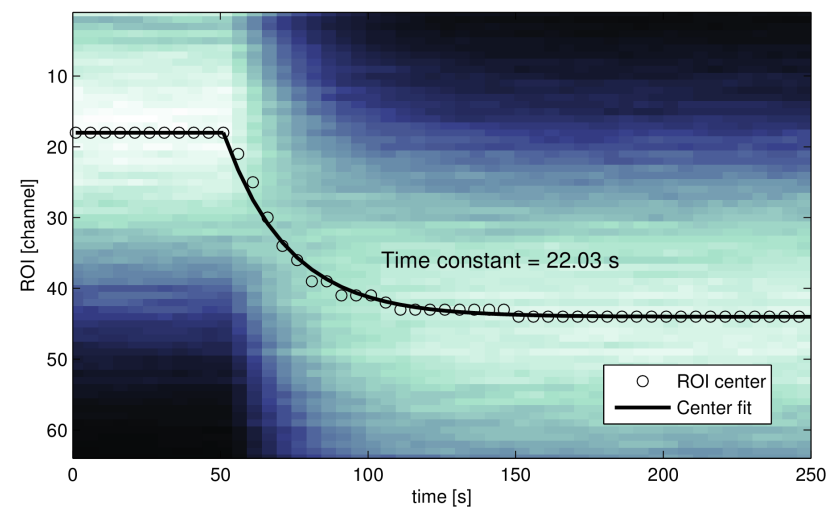

Fig. 3 Step response of the tracking algorithm, given an induced perturbation of the emission center of 26 channels. The ROI channels (background image) and center (o) were recorded using the communications module of the digital system. The fitted exponential (-) is an approximation to exponential response with a single time constant determined by the ROI area.

Although the algorithm involves several nonlinearities, its step response can be approximated as a single exponential, evidencing that the ROI area is the dominant factor in the dynamic of the system. The time constant can be fitted to 22.03 seconds, matching the value predicted from $N / m$ (21.84 seconds). This time constant can be used as an approximated limit of the operating point changes that can be compensated by the system.

\section{Conclusions}

The presented design involves a moderate amount of digital resources that can be appended as a module to an existing FPGA based acquisition systems, or implemented as a separate block. Inclusion of the tracking module does not affect performance of the existing digital processing module and can be configured, monitored, enabled or disabled on request during runtime.

Tracking the resonant energy level in Mössbauer experiments can drastically improve stability, hence performance, of data acquisition, especially in constantvelocity experiments where SCA long term output rate is a function of the parameter under study. Also, in constant-acceleration configurations, this technique relaxes HV stability requirements and room temperature control. 


\section{References}

1. Knoll, G.: Single-Channel Methods. In: Radiation Detection and Measurement, 3rd edition, pp. 685-690. John Willey \& Sons, New York (2000)

2. Ahmed, S.: Signal Processing. In: Physics and Engineering of Radiation Detection, pp. 463-520. Elsevier, London (2007)

3. Leo, W.R.: Electronics for Pulse Signal Processing. In: Techniques for Nuclear and Particle Physics Experiments, 2nd edition,, pp. 277-301. Springer-Verlag, Berlin (1994)

4. Veiga, A., Martínez, N., Mendoza Zélis, P., Pasquevich, G.A., Sánchez, F.H.: Advances in constant-velocity Mössbauer instrumentation. Hyperfine Interact. 167, 905-909 (2006)

5. Mendoza Zélis, P., Pasquevich, G.A., Sánchez, F.H., Veiga, A., Martínez, N.: A new application of Mössbauer effect thermal scans: determination of the magnetic hyperfine field temperature dependence. Phys. Lett. A 298, 55-59 (2002)

6. Pasquevich, G.A., Mendoza Zélis, P., Fernández van Raap, M.B., Sánchez, F.H.: Hyperfine field temperature dependence of Fe3Si from Mössbauer thermal scans. Physica B 354, 369-372 (2004)

7. Mendoza Zélis, P., Pasquevich, G.A., Veiga, A., Fernández van Raap, M.B., Sánchez, F.H.: A quasi-continuous observation of the $\alpha$-transition of $\mathrm{Fe} 1+\mathrm{xS}$ by Mössbauer line tracking. Hyperfine Interact. 195, 161-165 (2010)

8. Pasquevich, G.A., P. Mendoza Zélis,P., Sánchez, F. H., Fernández van Raap, M. B., Veiga, A., Martínez,N., Magnetic and thermal scans: A new Mössbauer effect approach, Hyperfine Interact., 167, 839-844 (2006)

9. Veiga, A., Pasquevich, G.A., Mendoza Zélis, P., Sánchez, F.H., Fernández van Raap, M.B., Martínez, N.: Experimental design and methodology for a new Mössbauer scan experiment: absorption line tracking. Hyperfine Interact. 188, 137-142 (2009)

10. V. Vanha-Honko, V.: The temperature dependence of the gas gain in sealed proportional counters. Nuclear Instruments and Methods 176, 213-219 (1980)

11. Sampietro, M.: A digital system for "optimum" resolution in x-ray spectroscopy Review of Scientific Instruments 66, 975-982 (1995)

12. Angelo Geraci, A.: Adaptive Digital Spectroscopy in Programmable Logic. IEEE Transactions on nuclear science 47, 2765-2772 (2000)

13. Roberto Abbiati, R: Self-configuring digital processor for on-line pulse analysis. IEEE Transactions on Nuclear Science 51, 826-830 (2004)

14. G. Pasquali, G: A DSP equipped digitizer for online analysis of nuclear detector signals.

Nuclear Instruments and Methods in Physics Research A 570, 126-132 (2007)

15. J. Pechousek, J.: Virtual instrumentation technique used in the nuclear digital signal processing system design: Energy and time measurement tests. Nuclear Instruments and Methods in Physics Research A 637, 200-205 (2011)

16. Nagy, S.: SCA window optimization on Mössbauer spectroscopy. J. Radioanal. Nucl. Chem. 137, 389-395 (1989)

17. Bravo, J.A.: Optimization Criteria in Mössbauer Spectroscopy. Hyperfine Interact. 148, 253261 (2003)

18. P. H. R. Scholefield, P.: Shift registers generating maximum-length sequences. Electronic Technology 37, 389-394 (1960)

19. Alfke, P.: Efficient Shift Registers, LFSR Counters, and Long Pseudo-Random Sequence Generators. Xilinx Application Note 052. http://www.xilinx.com/support/documentation/ /application_notes/xapp052.pdf (1996). Accessed 5 November 2012

20. Schönhage, A.: Finding the median. Journal of Computer and System Sciences 13, 184-199 (1976) 